LÚCia Alves da Silva Lara' ana Carolina Japur de Sá Rosa e Silva ${ }^{2}$ Adriana Peterson Mariano Salata Romão 3 Flavia Raquel Rosa Junquera ${ }^{4}$

\section{Abordagem das disfunções sexuais femininas}

\section{Artigo de Revisão}

Palavras-chave

Sexualidade

Disfunção sexual fisiológica/terapia Terapia de reposição hormonal Comportamento sexual

Keywords

Sexuality Sexual dysfunction, physiological/therapy Hormone replacement therapy Sexual behavior

\section{Resumo}

A disfunção sexual tem alta prevalência entre as mulheres. No entanto, os médicos raramente avaliam a vida sexual de suas pacientes, ou por se sentirem desconfortáveis em abordar a sexualidade ou porque desconhecem as técnicas de investigação. $\bigcirc$ modelo PILSET (PLISSIT), uma técnica de abordagem da função sexual humana, é composto por quatro elementos: permissão, informação limitada, sugestão específica e terapia sexual, que favorecem o diálogo entre o médico e a paciente, permitindo o acesso às queixas sexuais. A terapêutica consta de medidas de aconselhamento e orientações básicas sobre a função sexual, farmacoterapia e intervenções nos aspectos anátomo-funcionais do aparato sexual, com impacto positivo na vida sexual da mulher. Esta revisão mostra como usá-lo. Adicionalmente, vários aspectos da função sexual feminina, como prevalência, diagnóstico e outras modalidades de tratamento são discutidos.

\section{Abstract}

Sexual dysfunction prevalence is high among women. However, doctors rarely ask about their patients' sexual life, because they feel uncomfortable or because their knowledge about investigation techniques is insufficient. The PLISSIT model, a useful tool to access human sexual function, is composed by four elements: permission, limited information, specific suggestions, and intensive therapy, that favor dialogue between the doctor and the patient allowing the access to the sexual complaints. The therapeutics consists of counseling measures, drug prescription, basic orientations about sexual function and interventions on anatomic and functional aspects of the sexual apparatus with positive impact in the woman's sexual life. The present review shows how to use it. In addition, many aspects of female sexual dysfunction are discussed, such as prevalence, diagnostic and treatment options for female sexual dysfunction.
Departamento de Ginecologia e Obstetrícia da Faculdade de Medicina de Ribeirão Preto da Universidade de São Paulo - USP Ribeirão Preto (SP), Brasil.

'Coordenadora do Ambulatório de Estudos em Sexualidade Humana; Pós-graduanda do Departamento de Ginecologia e Obstetrícia da Faculdade de Medicina de Ribeirão Preto da Universidade de São Paulo - USP - Ribeirão Preto (SP), Brasil.

2 Doutora, Professora do Departamento de Ginecologia e Obstetrícia da Faculdade de Medicina de Ribeirão Preto da Universidade de São Paulo - USP - Ribeirão Preto (SP), Brasil.

${ }^{3}$ Pós-graduanda do Departamento de Ginecologia e Obstetrícia da Faculdade de Medicina de Ribeirão Preto da Universidade de São Paulo - USP - Ribeirão Preto (SP), Brasil.

${ }^{4}$ Médica Assistente do Departamento de Ginecologia e Obstetrícia da Faculdade de Medicina de Ribeirão Preto da Universidade de São Paulo - USP - Ribeirão Preto (SP), Brasil. 


\section{Introdução}

É cada vez mais reconhecida a importância da saúde sexual para a longevidade das relações afetivas e como parte da saúde global e bem-estar do indivíduo ${ }^{1,2}$. Atualmente, independente do gênero, o aspecto prazeroso do sexo tem demonstrado maior importância do que a sua finalidade reprodutiva ${ }^{2,3}$. Nos últimos dez anos, a mulher tem recorrido aos cuidados médicos, com mais freqüência, em busca de solução para os problemas que interferem na sua qualidade de vida, em especial aqueles relacionados com sua função sexual. No entanto, menos de $10 \%$ dos médicos têm a iniciativa de inquirir sobre as queixas sexuais de suas pacientes ${ }^{4,5}$. Em um estudo conduzido no Brasil, 4.753 ginecologistas responderam que a queixa de diminuição do desejo sexual estava entre os principais motivos de procura por consultas em seus consultórios ${ }^{6}$. Grande parte das mulheres admite que o ginecologista representa um papel fundamental no diagnóstico e manuseio das suas dificuldades sexuais, e gostariam que eles fossem mais qualificados nesta área ${ }^{5}$.

Por outro lado, alguns fatores limitam a prática da medicina sexual. Não obstante às altas taxas de disfunção sexual, grande parcela destas mulheres não busca ajuda médica, por vergonha, por frustração ou por falhas de tentativas de tratamento subprofissionalizado ${ }^{7}$. Uma minoria das mulheres tem a iniciativa de falar sobre suas dificuldades sexuais e apenas uma pequena parcela dos ginecologistas questiona sobre a função sexual de suas pacientes ${ }^{4}$. É possível que dificuldades pessoais do médico em relação à própria sexualidade restrinjam o seu acesso à sexualidade das pacientes. Em um estudo envolvendo 132 estudantes médicos com média de idade de 25 anos, a disfunção erétil e ejaculação rápida ocorriam em $28 \%$ dos homens. Entre mulheres, 32\% referiam desejo sexual hipoativo (DSH), 37\% referiam disfunção de orgasmo e $28 \%$ queixavam-se de insatisfação sexual $^{8}$. Embora a amostra seja limitada, serve de alerta para que este aspecto seja levado em conta na apreciação dos resultados da intervenção do médico na saúde sexual das pacientes. Outros fatores limitantes incluem a inexistência de pesquisas clínicas que evidenciem as taxas de resultados com intervenção em disfunções sexuais de origem orgânica, a inexistência de medicamento aprovado para o tratamento da disfunção sexual feminina e evidência insuficiente de dados sobre a eficácia de droga ou eficácia de intervenção psicológica? .

No Brasil, a sexologia é um campo novo dentro da especialidade de Ginecologia e Obstetrícia e possibilita a qualificação do médico para o acesso às estratégias de abordagem das queixas sexuais. Tornou-se um desafio criar uma plataforma de assistência básica que permita ao ginecologista diagnosticar a queixa sexual de fundo psíquico e biológico, e fazer a intervenção baseada em conhecimento sedimentado das diferentes especialidades médicas e afins ${ }^{10}$. Embora pareça complexo, a evolução da pesquisa em sexologia abordando amplamente o complexo biopsicosociocultural da função sexual humana disponibiliza um contingente sem precedentes de informações que possibilitam a construção de modelos de abordagem das disfunções sexuais de complexidade variável, adequáveis aos programas de assistência individual e populacional. No entanto, para otimizar a prática da medicina sexual na identificação e intervenção das queixas sexuais masculinas e femininas, são necessários ajustes consensuais que contemplem as diferenças culturais em todo o mundo ${ }^{11}$. Sendo assim, cada país necessita adequar seus protocolos de assistência, o que significa que não basta conhecer o "estado da arte" no âmbito mundial, uma vez que muitas estratégias de intervenção, aplicáveis em uma dada população, não apresentam resultados satisfatórios em culturas diferentes. Além disto, é preciso atentar para a necessidade de estratificação regional em uma mesma cultura ${ }^{11}$, principalmente em países de grandes dimensões territoriais.

Três conceitos devem ser sublinhados na prática da medicina sexual: adoção de uma estrutura centrada no paciente para avaliação e tratamento; aplicação dos princípios da medicina baseada em evidências para o planejamento do diagnóstico e do tratamento; e utilização de abordagem unificada para o homem e para a mulher. Em conjunto, estes três princípios constituem a abordagem balanceada e integrada para o tratamento da disfunção sexual $^{11}$. Considera-se que a história sexual e geral, história psicossocial, o exame físico geral e pélvico e testes laboratoriais são algoritmos comuns para o diagnóstico e planejamento da intervenção nas disfunções sexuais masculinas e femininas ${ }^{11,12}$, e a identificação dos fatores de risco como sendo fundamental para nortear estratégias de prevenção ${ }^{7}$.

O já consagrado modelo Permission, Limited Information, Specific Suggestions, Intensive Therapy $(\text { PLISSIT })^{13}$ ilustra o fato de que uma parcela expressiva dos problemas sexuais é passível de uma abordagem menos complexa e dispensa a terapia sexual intensiva. Isto porque muitas queixas sexuais são relativas à inadequação sexual e não à disfunção sexual. Este modelo, além de possibilitar a abordagem pelo ginecologista dos casos de menor complexidade (causas anatômicas, alguns distúrbios psíquicos, desconhecimento da anatomia), prevê a atuação do terapeuta sexual para os casos mais complexos. Este modelo tem sido considerado como o que mais atende à diversidade etiológica das queixas sexuais ${ }^{14-16}$.

Esta revisão tem como objetivos levantar as causas e prevalência das disfunções sexuais e oferecer estratégias de abordagem da função sexual feminina. Para isto, 
procedeu-se a uma busca no PubMed, no período de 1974 a 2008, por trials, coortes, estudos transversais, revisões de literatura e opiniões de especialistas em sexualidade, a fim de prover informações sobre as taxas de ocorrência das disfunções sexuais femininas e os fatores de risco primários para esta condição, bem como os fatores que dificultam a prática da sexologia. Tais informações norteiam o planejamento dos programas de intervenção e prevenção das disfunções sexuais que alcançam altas taxas de prevalência em todo o mundo ${ }^{17}$.

\section{Epidemiologia e fatores de risco}

\section{para disfunções sexuais}

Estima-se que entre 40 e $45 \%$ das mulheres e de 20 a $30 \%$ dos homens têm alguma queixa de disfunção sexual $^{18}$. Entre mulheres com queixas, a prevalência de DSH varia de 32 a $58 \%{ }^{19}$ e a disfunção de excitação e anorgasmia giram em torno de $30 \%{ }^{20}$. A dispareunia tem incidência variável e aumenta com o progredir da idade da mulher ${ }^{20}$. O DSH ocorre mais freqüentemente em mulheres em relacionamentos de longa duração ${ }^{20}$.

Em um estudo em uma população de mulheres na premenopausa, a queixa sexual mais comum foi o DSH (77\%), a disfunção de excitação ocorreu em $62 \%$ e a dificuldade para alcançar o orgasmo foi referida em $56 \%$ das pacientes? ${ }^{7}$.

A função sexual em idosos é pouco conhecida. Em um estudo conduzido com 74 pacientes com média de idade de 81 anos, $18 \%$ das mulheres e $41 \%$ dos homens permaneciam sexualmente ativos. As disfunções sexuais mais prevalentes em mulheres foram DSH, diminuição da lubrificação, anorgasmia e dispareunia, e a disfunção erétil ocorreu na maioria dos homens ${ }^{4}$.

O comprometimento do estado de saúde geral, doença cardiovascular e geniturinária, desordem psicológica e psiquiátrica, doenças crônicas, fatores relacionais e condições sociodemográficas desfavoráveis são fatores de risco associados com disfunção sexual para ambos os $\operatorname{sexos}^{18}$. Serão discutidos os mais relevantes e com maior potencial de exposição ao longo da vida da mulher.

\section{Fatores relacionais}

O DSH é a queixa mais comum em mulheres em relacionamentos de longa duração. Segundo Colson et al. ${ }^{3}$, embora na idade adulta, metade de homens e mulheres com parcerias refira pensar em sexo com freqüência. $\mathrm{Na}$ grande maioria das vezes é necessário que haja um estímulo para instigar o engajamento para o ato sexual entre o casal. Isto significa que a busca para a relação sexual partindo da predisposição instintiva, que é mais relacionada com a paixão, diminui com o avançar do relacionamento ${ }^{3,21}$. Estudos com ressonância magnética funcional evidenciam que as áreas cerebrais ativadas em mulheres apaixonadas são diferentes daquelas ativadas em mulheres em relacionamento de longa duração expostas ao estímulo do parceiro ${ }^{22}$. A expressão clínica deste achado é a queixa de falta de estímulo espontâneo para a relação sexual, interpretado como DSH. Com o decorrer do tempo, mesmo relacionamentos amorosos não conflituosos podem cursar com redução no interesse por sexo e diminuição ou perda da motivação pela interação sexual, em decorrência da inércia provocada pela rotina sexual que desmotiva a busca ${ }^{23}$. Este fato foi contemplado na última revisão da resposta sexual que inclui, como normal, a mulher ter múltiplas razões para se engajar no ato sexual, além da razão puramente instintiva ${ }^{24-26}$, e coloca dentro do padrão normal, as mulheres que necessitam de estímulo corporal direto pelo parceiro ou utilizam a fantasia sexual como estímulo para engajar na relação sexual ${ }^{25}$. As mulheres com maior prioridade para sexo e com bom relacionamento afetivo com seus parceiros mesmo em relacionamentos de longa duração, apresentam menos queixas de $\mathrm{DSH}^{20}$. Os conflitos relacionais têm impacto significante na função sexual ${ }^{27}$.

\section{Climatério e o progredir da idade}

Ocorre um aumento significativo das queixas sexuais relacionadas com o DSH, disfunção de orgasmo e dispareunia em mulheres nas fases de pré- e pós-menopausa ${ }^{28}$. O estudo longitudinal Assessment of Ageing in Women $(\mathrm{LAW})^{29}$, em 2004, investigou o comportamento sexual em mulheres australianas em uma faixa etária entre 40 a 49 e 70 a 79 anos, e observou que a procura pela relação sexual diminuiu notadamente com a idade. Comparando os dois grupos, a indiferença para freqüência sexual aumentou de $26,7 \%$ no primeiro grupo, para $72,3 \%$ entre as mais idosas ${ }^{29}$. No entanto, não se sabe ao certo se o incremento das disfunções sexuais nesta fase se deve à diminuição dos níveis hormonais ou ao progredir da idade $^{30}$. O envelhecimento cursa com alterações dos níveis hormonais, ação ineficiente dos neurotransmissores e neuropeptídeos, e redução tecidual dos fatores de crescimento e de outras substâncias envolvidas na função biológica ${ }^{31}$. O avançar da idade e as mudanças nos níveis hormonais no climatério podem ter repercussões biológicas e psíquicas negativas ${ }^{32}$ que são desfavoráveis aos sistemas envolvidos na resposta sexual normal ${ }^{33}$. A percepção do envelhecimento, o nível cultural, o grau de satisfação emocional com o parceiro e a lubrificação vaginal inadequada influenciam significativamente o desejo sexual e o orgasmo ${ }^{34}$.

Embora ocorra um incremento na disfunção sexual diretamente correlacionado com o aumento da idade ${ }^{35}$, parece que a angústia associada com a perda de desejo sexual diminui com o envelhecimento. Acredita-se que seja este um processo de adaptação biológica pré-programado ${ }^{36}$. 


\section{Disfunções sexuais do parceiro}

A procura para a relação sexual diminui notadamente com a idade por parte dos parceiros masculinos ${ }^{29}$. Em um estudo multicêntrico envolvendo 12.563 homens e mulheres na idade reprodutiva e idosos, $48 \%$ dos homens referiam algum grau de disfunção erétil e $63 \%$ das mulheres não estavam satisfeitas com o grau de ereção de seus parceiros ${ }^{1}$, com repercussões negativas no desejo sexual em ambos os sexos. Em um estudo conduzido na França em homens com média de idade de 35 anos, 25\% referiram diminuição do desejo sexual, $24 \%$ referiram ejaculação rápida e $15 \%$ referiram disfunção erétil ${ }^{3}$. A menor procura para o ato sexual por parte do parceiro pode ser interpretada como sinal de desamor e perda do potencial de sedução da mulher, com reflexo negativo em qualquer fase da resposta sexual ${ }^{27,37}$.

\section{Fatores psíquicos, estresse e patologias gerais}

Estados depressivos e distúrbios psíquicos podem cursar com disfunção sexual. As tensões no trabalho têm impacto negativo na função sexual, especialmente em mulheres ${ }^{3}$. Experiência sexual prévia negativa e traumas por violência sexual, como abuso sexual na infância e estupro, têm alto impacto negativo na função sexual ${ }^{35}$. No Brasil, embora não exista dado estatístico, calcula-se que sejam altas as cifras de abuso sexual contra criança ${ }^{38}$, o que significa que este precedente deve ser sempre considerado frente a uma paciente com queixa sexual. Doenças sistêmicas como diabetes $^{39}$, hiperprolactinemia ${ }^{40}$ e hipotireoidismo ${ }^{41}$ podem cursar com disfunção sexual.

\section{Uso de medicamentos}

Os anticoncepcionais orais promovem o aumento dos níveis séricos da SHBG (Sex Hormone Binding Globulin), a proteína transportadora dos esteróides sexuais. Esta proteína se liga aos androgênios, resultando em menor disponibilidade da testosterona livre, que é a fração androgênica ativa $^{42}$. Embora a participação dos androgênios na função sexual não esteja esclarecida, existem evidências de que a diminuição dos níveis séricos da testosterona livre pode cursar com disfunção sexual ${ }^{43}$. Os anticoncepcionais, em particular os de baixa concentração de estrogênios, podem causar diminuição da lubrificação vaginal e alterações no trofismo da parede vaginal, levando à dispareunia, com repercussões negativas na fase da excitação genital ${ }^{43-45}$.

Alguns antidepressivos, particularmente os inibidores da recaptação da serotonina ${ }^{46}$, largamente utilizados em todo o mundo, podem cursar também com disfunção sexual.

\section{Diagnóstico}

$\mathrm{O}$ acesso às disfunções sexuais pelo médico sem formação em terapia sexual deve se restringir às queixas que envolvem o desconhecimento da anatomia e da resposta sexual, e causas relacionadas a doenças ou condições fisiológicas que interferem na resposta.

Existem características especificas que diferenciam a resposta sexual feminina da masculina. A expressão da sexualidade masculina é centrada na conquista e posse e o ato sexual visa o orgasmo, enquanto que a expressão da sexualidade feminina se caracteriza pela sedução e entrega, sendo o desejo sexual o ponto principal da resposta sexual $^{12,47}$. Isto significa que mesmo mulheres que têm orgasmo durante a relação se queixam de disfunção sexual quando não sentem desejo ${ }^{47}$. Além disto, a resposta sexual feminina se caracteriza por alto grau de complexidade ${ }^{48}$, demonstrada no último modelo proposto por Basson et al. ${ }^{12}$, norteado na análise das múltiplas razões que levam a mulher a se engajar na relação sexual. Segundo este modelo, a mulher inicia a relação com ou sem consciência do desejo, ou porque é receptiva e responsiva a um estímulo erótico que resulta em excitação subjetiva com resposta física, ou porque tem excitação subjetiva que desencadeia a consciência do desejo que leva ao aumento gradativo da excitação e mais desejo. Este complexo de prazer e entrega pode culminar ou não em alívio orgástico, resultando em satisfação física e emocional que a torna receptiva para relações sexuais posteriores ${ }^{12}$. Portanto, é importante frisar que a mulher, mesmo não atingindo o orgasmo em uma relação sexual, pode se sentir totalmente satisfeita. Pode-se dizer que o marcador de satisfação sexual para a mulher é o desejo de engajar em nova relação com o parceiro, após um relacionamento sexual satisfatório, enquanto que o homem finaliza a resposta sexual com o orgasmo. Este desfecho contempla a finalidade procriativa do ato sexual e permite compreender que a relação sexual humana tem matizes desde um comportamento mais instintivo ao mais afetivo na busca do prazer.

A disfunção sexual se caracteriza pela alteração desde a manifestação instintiva até ausência de resposta ao estímulo e alterações nas fases do desejo, excitação e orgasmo ${ }^{49}$. A investigação se inicia com uma identificação cuidadosa da paciente por ser esta, com freqüência, o aspecto mais importante na gênese da patologia sexual. $\mathrm{O}$ avançar da idade pode cursar com afetação, principalmente, das fases do desejo e orgasmo ${ }^{50}$; a profissão pode gerar estresse e interferir com o desejo sexual; o nível socioeconômico e a cultura modulam a expressão sexual ${ }^{51}$. De maneira geral, a paciente queixa-se de não ter vontade de ter relação sexual. É necessário identificar a fase da resposta sexual afetada bem como estabelecer o tempo de ocorrência da disfunção, além de verificar se a queixa é associável a algum acontecimento. A história sexual pregressa permite o acesso à expressão sexual prévia positiva ou negativa e a identificação de medicamentos (antidepressivos, anticoncepcionais e outros) que possam afetar a resposta 
Quadro 1 - Anamnese sexual

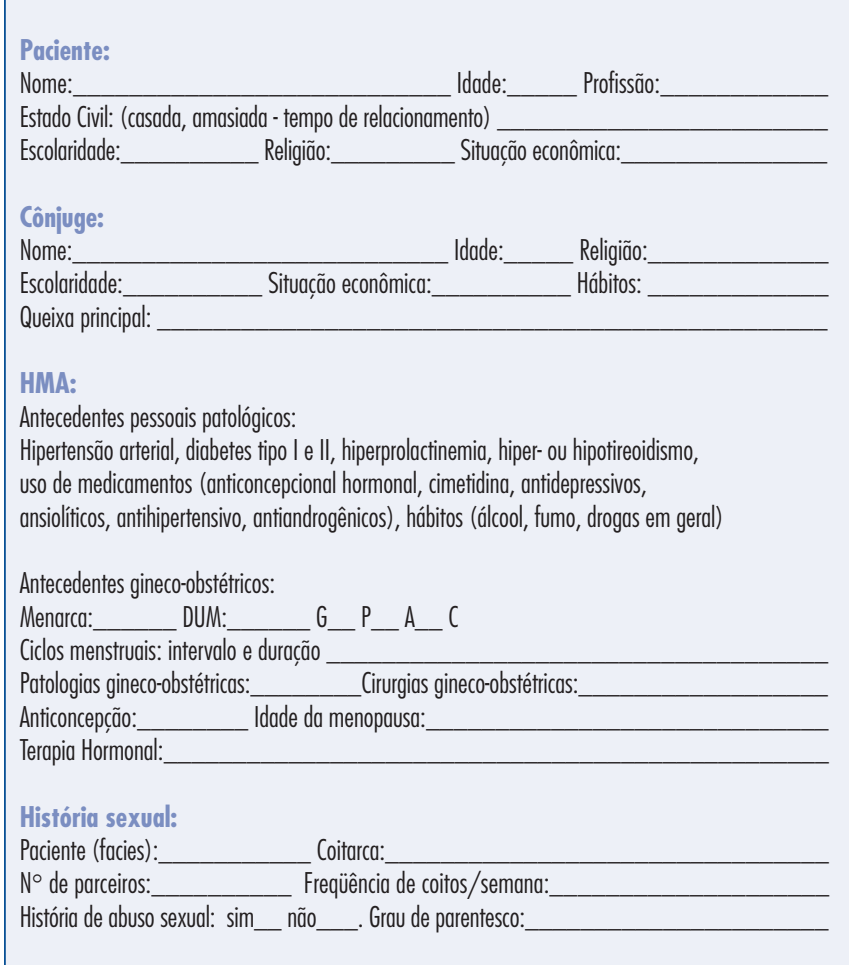

Quadro 2 - Classificação das disfunções sexuais segundo a Associação Psiquiátrica Americana

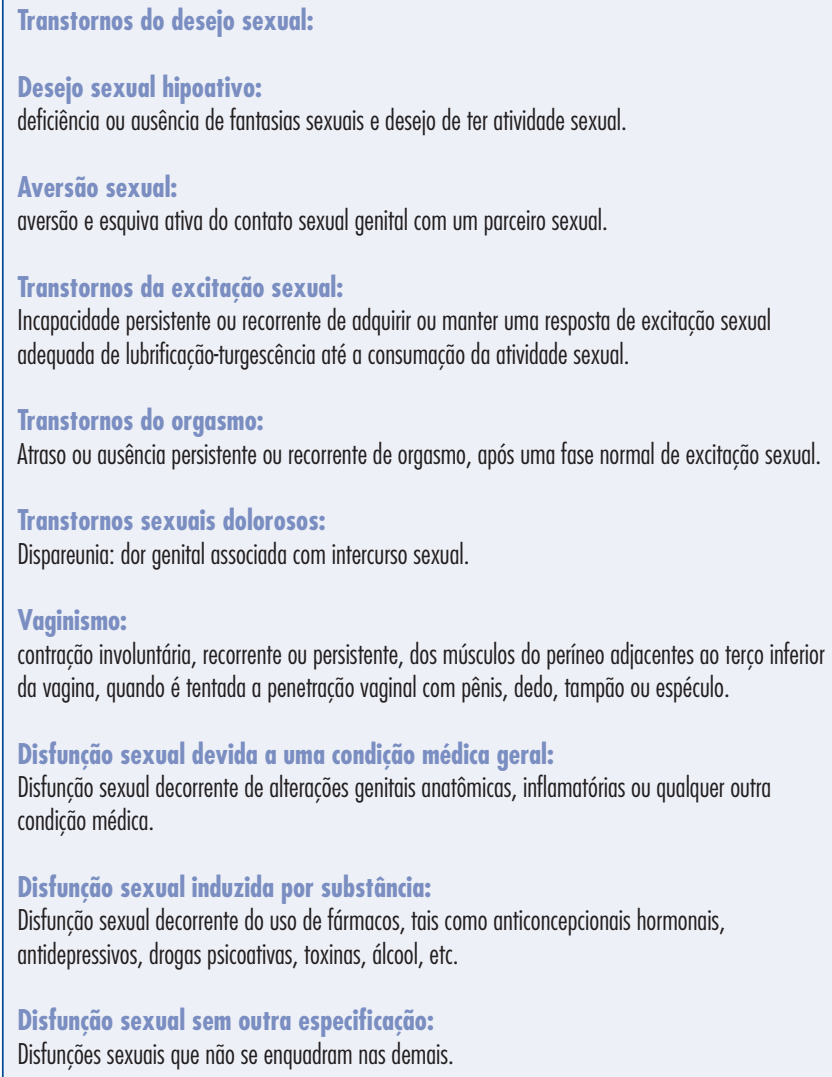

sexual $^{52}$. Doenças como diabetes mellitus, hipotireoidismo e hiperprolactinemias também podem cursar com disfunção sexual. Ao término da investigação, é importante ater-se para a fácies da paciente, porque o interrogatório sexual pode gerar desconforto e aflorar sentimentos negativos em relação à sexualidade. Tal situação requer a intervenção do psicoterapeuta. É importante conhecer a motivação da paciente para a busca do tratamento. Nem sempre a paciente procura ajuda para melhorar a sua qualidade de vida sexual: às vezes, o faz por imposição do parceiro, ou por medo de separação, ou ainda com o propósito de manter a família. A observância destes aspectos é fundamental para o sucesso do tratamento e para a avaliação do impacto da intervenção ${ }^{53,54}$. Em geral, a melhora das queixas sexuais é traduzida em mudança na aparência física, na atitude e na expressão facial da paciente ${ }^{54,55}$.

O Quadro 1 mostra um modelo de anamnese estruturada para a investigação sobre a função sexual.

O diagnóstico das disfunções sexuais é eminentemente clínico, mas justifica-se a dosagem da prolactina e TSH (Thyroid-Stimulating Hormone) em casos de DSH quando não está relacionado com uso de medicamentos ou fatores psíquicos ${ }^{56}$. A hiperprolactinemia pode cursar com disfunção sexual e afeta, principalmente, o desejo ${ }^{57}$. A dosagem de testosterona livre não tem utilidade porque o uso da testosterona para o tratamento das disfunções sexuais femininas permanece ainda controvertido.

Segundo a Associação Psiquiátrica Americana, as disfunções sexuais são classificadas em desejo sexual hipoativo, disfunção de excitação, anorgasmia, dispareunia e vaginismo ${ }^{58}$ (Quadro 2).

As disfunções sexuais são ainda classificadas em primária, quando a resposta sexual não alcança êxito ao longo da vida, secundária, quando é adquirida e situacional, quando a mulher apresenta disfunção somente na presença do parceiro ou em circunstância específicas ${ }^{59}$.

\section{Tratamento}

O tratamento das queixas sexuais envolve medidas gerais para o controle dos sintomas somáticos, psíquicos, locais e dificuldades com o parceiro e medidas específicas que contemplem as queixas individuais ${ }^{60}$.

O modelo PILSET (PLISSIT) é conciso e permite uma abordagem ampla envolvendo as queixas de origem biológica, psíquica e aquelas referentes ao desconhecimento da anatomia e da resposta sexual ${ }^{61}$, conforme descrição dos quatro elementos que o compõe: permissão (P): o profissional "permite" que a paciente tenha relação sexual considerando os aspectos fisiológicos da resposta sexual. Envolve, principalmente, a desmistificação e quebra de preconceitos. Por exemplo, o profissional "permite" que a paciente utilize fantasias sexuais ou filmes eróticos 
como estímulo sexual; informação limitada (IL): informar sobre a fisiologia da resposta sexual e sobre a anatomia da genitália, orientando os locais de maior sensibilidade prazerosa da vulva e vagina, que correspondem ao clitóris, pequenos lábios e intróito vaginal, sendo estas as áreas de maior conteúdo vásculo-nervoso ${ }^{62,63}$; sugestão específica (SE): aconselhar e sugerir mudanças na conduta sexual de acordo com critérios fundamentados na fisiologia da resposta sexual, e orientar mudança de atitude (atitude assertiva), se por exemplo: a paciente tem uma relação conflituosa com o parceiro, mas se mantém a relação, é porque tem razões pessoais para isto. $\mathrm{O}$ aspecto negativo $\mathrm{da}$ relação passa a ter mais peso do que o positivo, resultando em balanço desfavorável da relação afetivo-relacional. Quando a paciente atenta para os aspectos positivos do relacionamento pode ocorrer melhora da relação conjugal com repercussão positiva na vida sexual ${ }^{64}$; terapia sexual (T): encaminhar para a terapia sexual todos os casos de conflitos mais severos, como anedonia, conflitos relacionais seguidos de agressão verbal ou física, abuso sexual e distúrbios de comportamento ou desvio sexual (pedofilia, voyeurismo etc.) e outros, além daqueles que não respondem às orientações básicas.

As medidas gerais são consideradas estratégias de primeira linha em intervenção ${ }^{56}$ e destacam-se: a orientação sobre a anatomia da genitália e o esclarecimento sobre a resposta sexual humana, a prescrição de lubrificantes vaginais, a inclusão dos homens em programas de informação sobre sua função sexual e sobre a função sexual feminina ${ }^{34}$, a orientação sexual para a paciente e seu parceiro, modificação de causas reversíveis através do aconselhamento, mudança do estilo de vida, entre outras ${ }^{56}$. Tais medidas estão previstas dentro da abordagem proposta pelo modelo PLISSIT. Adicionalmente, ao médico cabe a intervenção na saúde geral e tratamento de doenças infecciosas do trato genital que possam cursar com dispareunia, bem como intervenções cirúrgicas para correção de incontinência urinária, prolapso genital, frouxidão vaginal, estenoses e outras que estejam associadas à queixa sexual da paciente ${ }^{65-67}$. Inclui também a substituição de medicamentos que interferem com a resposta sexual e prescrição de exercícios específicos para o fortalecimento da musculatura pélvica ${ }^{56}$.

Embora o papel dos esteróides sexuais na função sexual não esteja definido, a reposição oral parece favorecer a resposta sexual em mulheres climatéricas por melhorar os sintomas como irritabilidade, insônia e outros ${ }^{68-70}$. A diminuição da lubrificação vaginal culmina com queixas de dor coital. Em geral, o estrogênio ministrado por via oral atinge níveis ótimos em todas as camadas da parede vaginal, com resposta local adequada ${ }^{71}$. No entanto, se a queixa de secura vaginal persistir, faz-se necessária a complementação local do estrogênio ${ }^{72}$. Mulheres com queixas de desconforto na relação sexual, mas que não apresentam indicação para a reposição estrogênica oral, se beneficiam da prescrição do estrogênio tópico, que é suficiente para melhorar o trofismo vaginal ${ }^{73}$. Com o tratamento diário ocorre melhora do $\mathrm{pH}$, restabelecimento da flora e dos sintomas de secura vaginal e queimação, bem como dos sintomas urinários ${ }^{74}$. Embora não exista consenso quanto à duração da reposição estrogênica local, existem evidências de que a reposição de curto prazo seguida de intervalos é mais efetiva ${ }^{75}$, porque a constante exposição ao estrogênio promove a saturação dos seus receptores, resultando em resposta inadequada ${ }^{76,77}$. Não há indicação para a reposição oral visando unicamente a melhora dos sintomas genitais, tendo em vista a efetividade da via local genital ${ }^{78}$. Com a finalidade de melhora da resposta sexual, a terapia estrogênica oral fica restrita aos casos com disfunção, principalmente da fase de excitação ${ }^{79}$.

O tratamento para o câncer hormônio-dependente cursa com alterações importantes na vida sexual e na qualidade de vida da mulher ${ }^{80}$, mas a reposição estrogênica, mesmo local, é contra-indicada para estes casos ${ }^{81}$. As mulheres em tratamento de câncer de mama e endométrio devem fazer uso regular de lubrificantes vaginais ${ }^{82}$. Outra opção é o promestriene, que estimula a proliferação do epitélio vaginal sem efeito evidente no endométrio ${ }^{83}$.

Permanece controvertida a reposição androgênica para o tratamento das disfunções sexuais, tanto em relação à segurança quanto à eficácia ${ }^{84,85}$. Alguns autores preconizam a prescrição de androgênios para mulheres portadoras da síndrome da deficiência androgênica e para as mulheres submetidas a ooforectomia ${ }^{68,78}$. No entanto, o Endocrine Society Clinical Practice Guideline informa a inexistência de evidências suficientes para apoiar a recomendação do uso de androgênios e para atestar a segurança dos mesmos para o controle das disfunções sexuais femininas em geral $^{86}$. Porém, nos casos em que a reposição estroprogestínica não se mostra efetiva, alguns autores recomendam considerar a reposição androgênica conjunta ${ }^{87,88}$. Se a opção é a reposição oral, iniciar com $1,5 \mathrm{mg} / \mathrm{dia}$ de metiltestosterona. A dose deverá ser individualizada e não deve exceder $2,5 \mathrm{mg} / \mathrm{dia}^{87}$, o que evita os efeitos colaterais. Para uso local, o propionato de testosterona a $2 \%$ poderá ser utilizado diariamente ou em dias alternados por um período individualizado, porque não existe consenso sobre a duração do tratamento. São conhecidos os riscos $\mathrm{da}$ androgenioterapia em mulheres pelo potencial aumento dos níveis séricos do colesterol ${ }^{89}$. O lipidograma é obrigatório antes do início do tratamento e durante o seguimento.

A tibolona tem impacto positivo na resposta sexual e parece ser mais efetiva para as queixas de DSH e para as pacientes com queixas de disfunção orgástica ${ }^{79}$. 
A psicoterapia deve ser instituída sempre que houver preponderância de fatores psíquicos, em especial os casos relacionados a abuso sexual, relações conflituosas, distúrbios de comportamento, auto-estima rebaixada e depressão.

\section{Tratamento de situações específicas}

- Desejo sexual hipoativo conseqüente a fatores relacionais: falta de companheirismo, inabilidade nas carícias, conflitos relacionais, relação agressiva, mágoa, vingança, rotina relacional, disfunção sexual masculina (ex: ejaculação rápida). Tratamento: informar a paciente sobre diferenças de gênero (resposta sexual masculina e feminina). Orientar homem e mulher separadamente. Ressaltar a importância do diálogo para sensibilizar o parceiro para carícias rotineiras e jogos sexuais. Realçar a importância de atentar para os pontos positivos do parceiro (assertividade), pois a mulher com disfunção sexual de origem relacional tende a concentrar-se nos traços negativos do parceiro. Ressaltar a importância do ajuste do casal para o equilíbrio familiar. Estimular o uso de fantasias sexuais em casos de rotina relacional ${ }^{52}$. Encaminhar para a psicoterapia ou terapia de casal os casais com relação conflituosa e/ou agressiva.

- Repressão sexual: as disfunções sexuais por repressão religiosa, familiar ou pelo parceiro devem ser encaminhadas para a psicoterapia ou terapia sexual, mas o ginecologista poderá discutir aspectos básicos, como a racionalidade dos preceitos morais que cerceiam a expressão sexual; por exemplo, o auto-erotismo: parece haver uma "verdade coletiva" de que homens podem se masturbar e mulheres não. A repressão sexual religiosa é secular e requer uma abordagem cuidadosa baseada em evidências para que não incorra em questionamentos quanto à orientação religiosa da paciente ${ }^{90}$. Mas pode-se discutir a racionalidade da imagem punitiva de Deus em relação à expressão sexual ${ }^{52}$.

- Morbidades e medicamentos como causas de desejo sexual hipoativo: substituir medicamentos, se possível: hipotensores, cimetidina, anticoncepcional oral, antidepressivos, neurolépticos, tratar vaginites recorrentes, corrigir níveis hormonais e tratar estados depressivos. A bupropiona tem sido utilizada nos casos de DSH concomitante com a depressão, por interferir menos ${ }^{91}$ ou até mesmo por promover melhora da disfunção sexual ${ }^{92}$. Nos casos de desejo sexual hipoativo secundário ao uso de anticoncepcional hormonal, substituir pelos que contenham derivados da 19-nortestosterona ou considerar a substituição pelo método não hormonal ${ }^{43,93}$.
- Anorgasmia primária: deve-se à dificuldade de entrega, desconhecimento da anatomia, inabilidade do parceiro, dificuldade de concentração e ausência de auto-erotismo. $\mathrm{O}$ tratamento envolve: auto-manipulação associada à fantasia sexual, contração rítmica da musculatura pélvica e perivaginal, manipulação pelo parceiro ${ }^{94,95}$.

- Vaginismo: é uma síndrome psicofisiológica cuja característica fundamental é a contração involuntária, recorrente ou persistente, dos músculos do períneo adjacentes ao terço inferior da vagina. A contração pode variar desde leve, induzindo alguma tensão e desconforto à penetração, até severa, impedindo a penetração. É conseqüente a repressão sexual familiar, social e religiosa, culto à virgindade, medo de dor à relação, conflito psíquico profundo, experiência sexual previa negativa, abuso sexual na infância, estupro ${ }^{95-97}$. Quando o objetivo da paciente vagínica é concretizar o ato sexual, se a causa não estiver relacionada com grande trauma sexual em geral, é suficiente concentrar em relaxamento da musculatura perivaginal e técnicas de penetração, isto é, a mulher conduz a penetração. Estas pacientes, quando motivadas para a resolução, têm maior facilidade em se tocar do que as mulheres em geral, que carregam disfunção sexual de origem repressiva. Para o tratamento, fazer a dessensibilização lenta e progressiva, tocando a musculatura perineal e, com ordem verbal, orientar o relaxamento dos músculos que vão sendo palpados de forma gradativa. Após conseguir o relaxamento, fazer o toque somente unidigital. Informar que este toque não irá romper o hímen. Forçar a musculatura em direção ao ânus. Em casa, a paciente fará este exercício pela manhã e à noite. A paciente deverá segurar o pênis do parceiro e orientar a penetração. A toxina botulínica é indicada apenas para os casos com evolução desfavorável, mas este deverá ser um procedimento utilizável quando todos os outros recursos são falhos ${ }^{98}$. Se estas orientações não são suficientes, encaminhar para a terapia sexual e psicoterapia conjunta. É fundamental verificar se o parceiro não apresenta disfunção sexual, fato comum em associação com mulheres portadoras de vaginismo.

- Abuso sexual: todos os casos com história de abuso sexual deverão ser encaminhados à psicoterapia. Estes casos cursam, freqüentemente, com distúrbios psicológicos complexos ${ }^{99}$. Mas o ginecologista poderá oferecer apoio ao acolher e encaminhar. O ginecologista deve reconhecer, verbalmente, o peso do acontecimento para a paciente, mas deve 
tranqüilizá-la da possibilidade de resgate pleno da sua sexualidade desde que a paciente queira e se empenhe.

Em geral, as disfunções sexuais não ligadas a conflitos profundos (mágoa, vingança, estado depressivo, abuso sexual, estupro, outros) melhoram com a orientação sexual apropriada. Se as queixas permanecem, o ginecologista deverá encaminhar a paciente a um serviço especializado.

\section{Conclusões}

As disfunções sexuais femininas alcançam alta incidência em qualquer faixa etária e são subdiagnosticadas, ou porque as pacientes não se queixam devido à inibição ou porque o médico não investiga por constrangimento ou por desconhecimento da resposta sexual humana.

A farmacoterapia para disfunção sexual feminina é bastante restrita. A reposição estrogênica local melhora as queixas genitais em mulheres climatéricas, com impacto positivo na resposta sexual, e a racionalidade da terapia androgênica ainda não está estabelecida.
O médico tem condições de abordar a saúde sexual da mulher usando de sua autoridade profissional fundamentada no conhecimento da anatomia, farmacologia e do comportamento humano.

As patologias sexuais ao alcance do ginecologista são as que estão ligadas à falta de conhecimento da anatomia genital e da resposta sexual humana, a dor coital por afecções do trato genital e a disfunção sexual conseqüente à depressão, e a patologias sistêmicas como diabetes, hipotireoidismo, hiperprolactinemias e outras.

As condições que devem ser encaminhadas à terapia sexual associada à psicoterapia, são aquelas associadas a forte repressão sexual e à violência sexual, e os casos que não respondem à intervenção médica.

O modelo PILSET (PLISSIT), composto por quatro elementos (permissão, informação limitada, sugestão específica e terapia sexual), oferece condições para o acesso das queixas sexuais e tem impacto positivo na função sexual através da utilização de técnicas de aconselhamento e orientações básicas sobre o aparato psíquico e biológico que controla a resposta sexual humana.

\section{Referências}

1. Mulhall J, King R, Glina S, Hvidsten K. Importance of and satisfaction with sex among men and women worldwide: results of the global better sex survey. J Sex Med. 2008;5(4):788-95.

2. Studd J. A comparison of 19 th century and current attitudes to female sexuality. Gynecol Endocrinol. 2007;23(12):673-81.

3. Colson MH, Lemaire A, Pinton P, Hamidi K, Klein P. Sexual behaviors and mental perception, satisfaction and expectations of sex life in men and women in France. J Sex Med. 2006;3(1):121-31.

4. Smith L, Mulhall JP, Deveci S, Monaghan N, Reid MC. Sex after seventy: a pilot study of sexual function in older persons. J Sex Med. 2007;4(5): 1247-53.

5. Martinez L. More education in the diagnosis and management of sexual dysfunction is needed. Fertil Steril. 2008;89(4):1035.

6. Abdo CHN, Oliveira Junior WM. O ginecologista brasileiro frente às queixas sexuais femininas: um estudo preliminar. RBM Rev Bras Med. 2002;59(3): 179-86.

7. Berman L, Berman J, Felder S, Pollets D, Chhabra S, Miles M, et al. Seeking help for sexual function complaints: what gynecologists need to know about the female patient's experience. Fertil Steril. 2003;79(3):572-6.

8. Shindel AW, Ferguson GG, Nelson CJ, Brandes SB. The sexual lives of medical students: a single institution survey. J Sex Med. 2008;5(4):796-803.

9. Heiman JR, Guess MK, Connell K, Melman A, Hyde JS, Segraves $R T$, et al. Standards for clinical trials in sexual dysfunctions of women: research designs and outcomes assessment. J Sex Med. $2004 ; 1(1): 92-7$.
10. Wagner G. Sexual medicine in the medical curriculum. Int J Androl. 2005;28 Suppl 2:7-8.

11. Hatzichristou D, Rosen RC, Broderick G, Clayton A, Cuzin B, Derogatis $\mathrm{L}$, et al. Clinical evaluation and management strategy for sexual dysfunction in men and women. J Sex Med. 2004;1 (1):49-57.

12. Basson R, Althof S, Davis S, Fugl-Meyer K, Goldstein I, Leiblum S, et al. Summary of the recommendations on sexual dysfunctions in women. J Sex Med. 2004; 1 (1):24-34.

13. Annon J. Behavioral treatment of sexual problems. New York: Harper \& Row; 1976.

14. Frank JE, Mistretta P, Will J. Diagnosis and treatment of female sexual dysfunction. Am Fam Physician. 2008;77(5):635-42.

15. Taylor B, Davis S. Using the extended PLISSIT model to address sexual healthcare needs. Nurs Stand. 2006;21 (1 1):35-40.

16. Vauth $R$, Härter $M$, Hohagen $F$, Kemmerich $C$, Herrmann JM, Haag $G$, et al. Management of mental health and primary care. Development and evaluation of a training program based on the PLISSIT approach. Nervenarzt. 1999;70(1):54-63.

17. Derogatis LR, Burnett AL. The epidemiology of sexual dysfunctions. J Sex Med. 2008;5(2):289-300.

18. Lewis RW, Fugl-Meyer KS, Bosch R, Fugl-Meyer AR, Laumann EO, Lizza $E$, et al. Epidemiology/risk factors of sexual dysfunction. J Sex Med. 2004;1 (1):35-9.

19. Hayes RD, Dennerstein L, Bennett CM, Fairley CK. What is the "true" prevalence of female sexual dysfunctions and does the way we assess these conditions have an impact? J Sex Med. 2008;5(4):777-87. 
20. Hayes RD, Dennerstein L, Bennett CM, Sidat M, Gurrin LC, Fairley CK. Risk factors for female sexual dysfunction in the general population: exploring factors associated with low sexual function and sexual distress. J Sex Med. 2008;5(7):1681-93.

21. Fisher HE, Aron A, Mashek D, Li H, Brown LL. Defining the brain systems of lust, romantic attraction, and attachment. Arch Sex Behav. 2002;31(5):413-9.

22. Bartels A, Zeki S. The neural basis of romantic love. Neuroreport. 2000; 11 (17):3829-34.

23. Fisher $\mathrm{H}$, Aron A, Brown LL. Romantic love: an fMRI study of a neural mechanism for mate choice. J Comp Neurol. 2005;493(1):58-62.

24. Basson R. The complexities of female sexual arousal disorder: potential role of pharmacotherapy. World J Urol. 2002;20(2):1 19-26.

25. Basson R, Leiblum S, Brotto L, Derogatis L, Fourcroy J, Fugl-Meyer $\mathrm{K}$, et al. Revised definitions of women's sexual dysfunction. J Sex Med. 2004; 1(1):40-8.

26. Basson R, Brotto LA, Laan E, Redmond G, Utian WH. Assessment and management of women's sexual dysfunctions: problematic desire and arousal. J Sex Med. 2005;2(3):291-300.

27. Dennerstein L, Dudley E, Burger H. Are changes in sexual functioning during midlife due to aging or menopause? Fertil Steril. $2001 ; 76(3): 456-60$

28. Hisasue S, Kumamoto Y, Sato Y, Masumori N, Horita H, Kato $R$, et al. Prevalence of female sexual dysfunction symptoms and its relationship to quality of life: a Japanese female cohort study. Urology. 2005;65(1):143-8.

29. Howard JR, O'Neill S, Travers C. Factors affecting sexuality in older Australian women: sexual interest, sexual arousal, relationships and sexual distress in older Australian women. Climacteric. 2006;9(5):355-67.

30. Bretschneider JG, McCoy NL. Sexual interest and behavior in healthy 80- to 102-year-olds. Arch Sex Behav. 1988;17(2):109-29.

31. Garcia-Segura LM, Diz-Chaves $Y$, Perez-Martin M, Darnaudéry $M$. Estradiol, insulin-like growth factor-l and brain aging. Psychoneuroendocrinology. 2007;32 Suppl 1:S57-61.

32. Rosa e Silva ACJS, Sá MFS. Efeitos dos esteróides sexuais sobre o humor e a cognição. Rev Psiquiatr Clin (São Paulo). 2006;33(2):60-7.

33. Stotland NL. Menopause: social expectations, women's realities. Arch Womens Ment Health. 2002;5(1):5-8.

34. González M, Viáfara G, Caba F, Molina T, Ortiz C. Libido and orgasm in middle-aged woman. Maturitas. 2006;53(1):1-10.

35. Beutel ME, Stöbel-Richter Y, Brähler E. Sexual desire and sexual activity of men and women across their lifespans: results from a representative German community survey. BJU Int. 2008;101(1):76-82.

36. Graziottin A. Prevalence and evaluation of sexual health problemsHSDD in Europe. J Sex Med. 2007;4 Suppl 3:211-9.

37. Leiblum SR, Koochaki PE, Rodenberg CA, Barton IP, Rosen RC. Hypoactive sexual desire disorder in postmenopausal women: US results from the Women's International Study of Health and Sexuality (WISHeS). Menopause. 2006;13(1):46-56.

38. No authors listed. Child prostitution: family disintegration, necessary planning, the laziness of the elite. Planej Agora. 1993;9(239):4.

39. Grandjean C, Moran B. The impact of diabetes mellitus on female sexual well-being. Nurs Clin North Am. 2007;42(4):581-92; vi.

40. Kadioglu P, Yalin AS, Tiryakioglu O, Gazioglu N, Oral G, Sanli $O$, et al. Sexual dysfunction in women with hyperprolactinemia: a pilot study report. J Urol. 2005; 174(5):1921-5.

41. Zárate A, Basurto L, Hernández M. Thyroid malfunction in women. Ginecol Obstet Mex. 2001;69:200-5.
42. Graham CA, Bancroft J, Doll HA, Greco T, Tanner A. Does oral contraceptive-induced reduction in free testosterone adversely affect the sexuality or mood of women? Psychoneuroendocrinology. 2007;32(3):246-55.

43. Caruso S, Agnello C, Intelisano G, Farina M, Di Mari L, Cianci A. Sexual behavior of women taking low-dose oral contraceptive containing 15 microg ethinylestradiol/60 microg gestodene. Contraception. 2004;69(3):237-40.

44. Foster DC, Hasday JD. Elevated tissue levels of interleukin-1 beta and tumor necrosis factor-alpha in vulvar vestibulitis. Obstet Gynecol. 1997;89(2):291-6

45. Greenstein A, Ben-Aroya Z, Fass O, Militscher I, Roslik Y, Chen $\mathrm{J}$, et al. Vulvar vestibulitis syndrome and estrogen dose of oral contraceptive pills. J Sex Med. 2007;4(6): 1679-83.

46. Zemishlany $Z$, Weizman $A$. The impact of mental illness on sexual dysfunction. Adv Psychosom Med. 2008;29:89-106.

47. Nobre PJ, Pinto-Gouveia J. Cognitive and emotional predictors of female sexual dysfunctions: preliminary findings. J Sex Marital Ther. 2008;34(4):325-42.

48. Sand M, Fisher WA. Women's endorsement of models of female sexual response: the nurses' sexuality study. J Sex Med. 2007;4(3):708-19.

49. Clayton $\mathrm{AH}$. Epidemiology and neurobiology of female sexual dysfunction. J Sex Med. 2007;4 Suppl 4:260-8.

50. Abdo CH, Oliveira WM Jr, Moreira ED Jr, Fittipaldi JA. Prevalence of sexual dysfunctions and correlated conditions in a sample of Brazilian women-results of the Brazilian study on sexual behavior (BSSB). Int J Impot Res. 2004; 16(2): 160-6.

51. DeLamater J, Friedrich WN. Human sexual development. J Sex Res. 2002;39(1):10-4.

52. Junqueira FRR, Lara LAS, Romão APMS, Rosa e Silva ACJS, Romão GS, Ferriani RA. Implantação de um ambulatório de sexualidade em um serviço de ginecologia de hospital universitário: resultados após um ano. Reprod Clim. 2005;20:13-6.

53. Smith WJ, Beadle K, Shuster EJ. The impact of a group psychoeducational appointment on women with sexual dysfunction. Am J Obstet Gynecol. 2008; 198(6):697.el-6.

54. Althof SE, Leiblum SR, Chevret-Measson M, Hartmann U, Levine SB, McCabe $M$, et al. Psychological and interpersonal dimensions of sexual function and dysfunction. J Sex Med. 2005;2(6):793-800.

55. Hartman LM. Effects of sex and marital therapy on sexual interaction and marital happiness. J Sex Marital Ther. 1983;9(2):137-51 .

56. Goldstein I. Current management strategies of the postmenopausal patient with sexual health problems. J Sex Med. 2007;4 Suppl 3:235-53

57. Brunelleschi S, Zeppegno P, Risso F, Cattaneo Cl, Torre E. Risperidoneassociated hyperprolactinemia: evaluation in twenty psychiatric outpatients. Pharmacol Res. 2003;48(4):405-9.

58. Basson R, Berman J, Burnett A, Derogatis L, Ferguson D, Fourcroy J, et al. Report of the international consensus development conference on female sexual dysfunction: definitions and classifications. J Urol. 2000; 163(3):888-93.

59. Kaplan HS. A nova terapia do sexo. Rio de Janeiro: Nova Fronteira; 1974.

60. Mishra G, Kuh D. Sexual functioning throughout menopause: the perceptions of women in a British cohort. Menopause. 2006; 13(6):880-90.

61. Dixon KD, Dixon PN. The PLISSIT Model: care and management of patients' psychosexual needs following radical surgery. Lippincotts Case Manag. 2006; 11 (2):101-6. 
62. Levin RJ. VIP, vagina, clitoral and periurethral glans-an update on human female genital arousal. Exp Clin Endocrinol. 1991;98(2):61-9.

63. Pessina MA, Hoyt RF Jr, Goldstein I, Traish AM. Differential effects of estradiol, progesterone, and testosterone on vaginal structural integrity. Endocrinology. 2006;147(1):61-9.

64. Delehanty R. Changes in assertiveness and changes in orgasmic response occurring with sexual therapy for preorgasmic women. J Sex Marital Ther. 1982;8(3):198-208.

65. Cohen BL, Barboglio P, Gousse A. The impact of lower urinary tract symptoms and urinary incontinence on female sexual dysfunction using a validated instrument. J Sex Med. 2008;5(6):1418-23.

66. Bradway C, Strumpf N. Seeking care: women's narratives concerning long-term urinary incontinence. Urol Nurs. 2008;28(2):123-9.

67. Voorham-van der Zalm PJ, Lycklama A Nijeholt GA, Elzevier HW, Putter H, Pelger RC. "Diagnostic investigation of the pelvic floor": a helpful tool in the approach in patients with complaints of micturition, defecation, and/or sexual dysfunction. J Sex Med. 2008;5(4):864-71.

68. Kingsberg S, Shifren J, Wekselman K, Rodenberg C, Koochaki P, Derogatis L. Evaluation of the clinical relevance of benefits associated with transdermal testosterone treatment in postmenopausal women with hypoactive sexual desire disorder. J Sex Med. 2007;4(4 Pt 1):1001-8.

69. Gregersen N, Jensen PT, Giraldi AE. Sexual dysfunction in the peri- and postmenopause. Status of incidence, pharmacological treatment and possible risks. A secondary publication. Dan Med Bull. 2006;53(3):349-53.

70. Nelson HD. Menopause. Lancet. 2008;371(9614):760-70.

71. Tourgeman DE, Slater CC, Stanczyk FZ, Paulson RJ. Endocrine and clinical effects of micronized estradiol administered vaginally or orally. Fertil Steril. $2001 ; 75(1): 200-2$.

72. Suckling J, Lethaby A, Kennedy R. Local oestrogen for vaginal atrophy in postmenopausal women. Cochrane Database Syst Rev. 2003(4):CD001500.

73. Barentsen R, van de Weijer PH, Schram JH. Continuous low dose estradiol released from a vaginal ring versus estriol vaginal cream for urogenital atrophy. Eur J Obstet Gynecol Reprod Biol. $1997 ; 71(1): 73-80$.

74. Galhardo CL, Soares JM Jr, Simões RS, Haidar MA, Rodrigues de Lima $\mathrm{G}$, Baracat EC. Estrogen effects on the vaginal $\mathrm{pH}$, flora and cytology in late postmenopause after a long period without hormone therapy. Clin Exp Obstet Gynecol. 2006;33(2):85-9.

75. Gorodeski GI. Aging and estrogen effects on transcervicaltransvaginal epithelial permeability. J Clin Endocrinol Metab. $2005 ; 90(1): 345-51$

76. Gorodeski GI, Pal D. Involvement of estrogen receptors alpha and beta in the regulation of cervical permeability. Am J Physiol Cell Physiol. 2000;278(4):C689-96.

77. Gorodeski GI. Vaginal-cervical epithelial permeability decreases after menopause. Fertil Steril. 2001;76(4):753-61.

78. North American Menopause Society. The role of local vaginal estrogen for treatment of vaginal atrophy in postmenopausal women: 2007 position statement of The North American Menopause Society. Menopause. 2007; 14(3 Pt 1):355-69.

79. Cayan F, Dilek U, Pata O, Dilek S. Comparison of the effects of hormone therapy regimens, oral and vaginal estradiol, estradiol + drospirenone and tibolone, on sexual function in healthy postmenopausal women. J Sex Med. 2008;5(1):132-8.

80. Conde DM, Pinto-Neto AM, Freitas Júnior R, Aldrighi JM. Qualidade de vida de mulheres com câncer de mama. Rev Bras Ginecol Obstet. 2006;28(3): 195-204.
81. Derzko C, Elliott S, Lam W. Management of sexual dysfunction in postmenopausal breast cancer patients taking adjuvant aromatase inhibitor therapy. Curr Oncol. 2007;14 Suppl 1:S20-40.

82. Johnston SL, Farrell SA, Bouchard C, Farrell SA, Beckerson LA, Comeau M; SOGC Joint Committee-Clinical Practice Gynaecology and Urogynaecology. The detection and management of vaginal atrophy. J Obstet Gynaecol Can. 2004;26(5):503-15.

83. Borrelli AL, Casolaro AM, Esposito G, Berlingieri D. Biologic action of promestriene on the genital tract of the castrated rat. Minerva Ginecol. 1990;42(1 1):467-72.

84. Traish A, Guay AT, Spark RF; Testosterone Therapy in Women Study Group. Are the Endocrine Society's Clinical Practice Guidelines on Androgen Therapy in Women misguided? A commentary. J Sex Med. 2007;4(5):1223-34.

85. Braunstein GD. Management of female sexual dysfunction in postmenopausal women by testosterone administration: safety issues and controversies. J Sex Med. 2007;4(4 Pt 1):859-66.

86. Wierman ME, Basson R, Davis SR, Khosla S, Miller KK, Rosner W, et al. Androgen therapy in women: an Endocrine Society Clinical Practice guideline. J Clin Endocrinol Metab. 2006;91 (10):3697-710.

87. de Paula FJ, Soares JM Jr, Haidar MA, de Lima GR, Baracat EC. The benefits of androgens combined with hormone replacement therapy regarding to patients with postmenopausal sexual symptoms. Maturitas. 2007;56(1):69-77.

88. Basson R. Hormones and sexuality: current complexities and future directions. Maturitas. 2007;57(1):66-70.

89. Alexanderson C, Eriksson E, Stener-Victorin E, Lystig T, Gabrielsson $B$, Lönn $M$, et al. Postnatal testosterone exposure results in insulin resistance, enlarged mesenteric adipocytes, and an atherogenic lipid profile in adult female rats: comparisons with estradiol and dihydrotestosterone. Endocrinology. 2007;148(1 1):5369-76.

90. Hanly MA. Submission, inhibition and sexuality: masochistic character and psychic change in Austen's Mansfield Park. Int J Psychoanal. 2005;86(Pt 2):483-501.

91. Clayton $A H$, Croft HA, Horrigan JP, Wightman DS, Krishen A, Richard NE, et al. Bupropion extended release compared with escitalopram: effects on sexual functioning and antidepressant efficacy in 2 randomized, double-blind, placebo-controlled studies. J Clin Psychiatry. 2006;67(5):736-46.

92. Dobkin RD, Menza M, Marin H, Allen LA, Rousso R, Leiblum SR. Bupropion improves sexual functioning in depressed minority women: an open-label switch study. J Clin Psychopharmacol. 2006;26(1):21-6.

93. Bancroff J, Sartorius N. The effects of oral contraceptives on wellbeing and sexuality. Oxf Rev Reprod Biol. 1990; 12:57-92.

94. Buffat J. Why women consult sexologists: an approach for practicing physicians. Rev Med Suisse. 2005; 1 (1 1):754-6, 759-61.

95. Meston CM, Levin RJ, Sipski ML, Hull EM, Heiman JR. Women's orgasm. Annu Rev Sex Res. 2004; 15:173-257.

96. Badran W, Moamen N, Fahmy I, El-Karaksy A, Abdel-Nasser TM, Ghanem $\mathrm{H}$. Etiological factors of unconsummated marriage. Int J Impot Res. 2006; 18(5):458-63.

97. Reissing ED, Binik YM, Khalifé S, Cohen D, Amsel R. Etiological correlates of vaginismus: sexual and physical abuse, sexual knowledge, sexual self-schema, and relationship adjustment. J Sex Marital Ther. 2003;29(1):47-59.

98. Ghazizadeh S, Nikzad M. Botulinum toxin in the treatment of refractory vaginismus. Obstet Gynecol. 2004; 104(5 Pt 1):922-5.

99. Fitzgerald MM, Schneider RA, Salstrom S, Zinzow HM, Jackson J, Fossel RV. Child sexual abuse, early family risk, and childhood parentification: pathways to current psychosocial adjustment. J Fam Psychol. 2008;22(2):320-4. 